\title{
Nicht resorbierbares Antibiotikum verbessert Prognose der Leberzirrhose
}

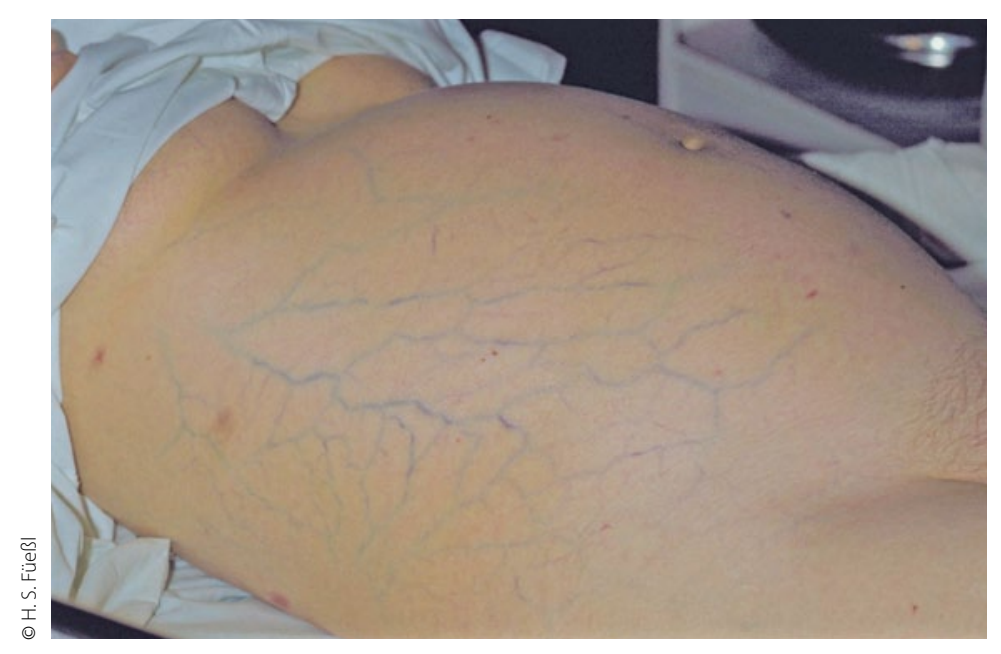

Schwerwiegende Komplikationen der Leberzirrhose wie dieser Aszites lassen sich mit einem nicht resorbierbaren Antibiotikum verhindern.
Die langfristige Darmdekontamination mit dem nicht resorbierbaren Antibiotikum Rifaximin verringert bei Patienten mit alkoholischer Leberzirrhose die Komplikationen der portalen Hypertension und erhöht die Überlebensrate.

— Die portale Hypertension bei der alkoholischen Leberzirrhose geht mit einer Reihe von schwerwiegenden Komplikationen einher wie Aszites, Ösophagusvarizenblutungen, hepatischer Enzephalopathie, spontaner bakterieller Peritonitis und dem hepatorenalen Syndrom. Für die Ätiopathogenese eines Teils dieser Zustände werden die toxischen Produkte von Darmbakterien verantwortlich gemacht. Daher besteht seit langem eines der Therapieprinzipien darin, den Darm weitgehend zu dekontaminieren.

In Kurzzeituntersuchungen konnte gezeigt werden, dass das neue, nicht resorbierbare Antibiotikum Rifaximin die Hämodynamik der Leber und die hepatische Enzephalopathie verbessert. In einer Fall-Kontroll-Studie hat man $23 \mathrm{~Pa}$ tienten mit einem Child-Pugh-Score von $>7$ und Aszites, die in den vorange- gangenen sechs Monaten alkoholabstinent waren, langfristig mit täglich $3 \times 400$ mg Rifaximin behandelt. Jedem dieser Patienten wurden zwei vergleichbare Kontrollpatienten gegenübergestellt und alle Patienten bis zu einer maximalen Zeitdauer von fünf Jahren nachbeobachtet.

Im Laufe dieser Zeit zeigte sich, dass die langfristig mit Rifaximin behandelten Patienten ein signifikant niedriges Risiko für die Entwicklung einer Varizenblutung ( $35 \%$ vs. 59,5\%), einer hepatischen Enzephalopathie (31,5\% vs. $47 \%)$, einer spontanen bakteriellen Peritonitis $(4,5 \%$ vs. $46 \%)$ und eines hepatorenalen Syndroms (4,5\% vs. $51 \%$ ) als die Kontrollen aufwiesen. Die kumulative 5-Jahres-Überlebenswahrscheinlichkeit der mit Rifaximin behandelten $\mathrm{Pa}$ tienten betrug $61 \%$ gegenüber nur 13,5\% der Kontrollpatienten. Eine multivariate Analyse ergab, dass die Anwendung von Rifaximin einen unabhängigen protektiven Faktor für die genannten Komplikationen darstellt.

\footnotetext{
- J. Vlachogiannakos et al.

(Korres.: jvlachog@hotmail.com): Long-term administration of rifaximin improves the prognosis of patients with decompensated alcoholic cirrhosis. J Gastroenterol Hepatol.2013; 28:450-455
}

\section{Kommentar}

Obwohl das Ergebnis der Studie eindrucksvoll ist, ist bis heute nicht bekannt, welche Wirkungen von Rifaximin genau dazu führen. In der Diskussion steht eine positive Beeinflussung der Leberhämodynamik infolge einer veränderten endotoxininduzierten Ausschüttung von Entzündungsmediatoren wie die TNF-alpha, IL-1 und IL-6. Serielle Messungen von Endotoxin und Zytokinen ergaben in der vorliegenden Studie allerdings kein vernünftiges Ergebnis, da die Werte bei beiden Gruppen von Tag zu Tag starken Schwankungen unterlagen, ohne dass sich dafür aufgrund des klinischen Zustandes eine Erklärung ergab.

Die Situation, wonach eine Therapie sehr erfolgreich ist, man aber ihre genaue Wirkungsweise nicht kennt, empfinden die meisten Ärzte als etwas unbefriedigend. Die ausgeprägten Unterschiede, insbesondere bei der spontanen bakteriellen Peritonitis und dem hepatorenalen Syndrom, sind aber bei aller methodischen Kritik an einer Fall-Kontroll-Studie so überzeugend, dass das Fehlen einer griffigen Hypothese zur Wirkung kein Hindernis für den Einsatz von Rifaximin bei Patienten mit alkoholischer Leberzirrhose und Aszites sein sollte.

H. S. FÜESSL 